\title{
Innate responses of the predatory mite Phytoseiulus persimilis to a herbivore-induced plant volatile
}

\author{
B. Sznajder $\cdot$ M. W. Sabelis $\cdot$ M. Egas
}

Received: 10 June 2010/ Accepted: 1 February 2011/Published online: 15 February 2011

(C) The Author(s) 2011. This article is published with open access at Springerlink.com

\begin{abstract}
The responses of the predatory mite $P$. persimilis to herbivore-induced plant volatiles are at least partly genetically determined. Thus, there is potential for the evolution of this behaviour by natural selection. We tested whether distinct predator genotypes with contrasting responses to a specific herbivore-induced plant volatile, i.e. methyl salicylate $(\mathrm{MeSa})$, could be found in a base population collected in the field (Sicily). To this end, we imposed purifying selection on individuals within iso-female lines of $P$. persimilis such that the lines were propagated only via the individual that showed either a preference or avoidance of MeSa. The responses of the lines were characterized as the mean proportion of individuals choosing MeSa when given a choice between MeSa and clean air. Significant variation in predator responses was detected among iso-female lines, thus confirming the presence of a genetic component for this behaviour. Nevertheless, we did not find a significant difference in the response to MeSa between the lines that were selected to avoid $\mathrm{MeSa}$ and the lines selected to prefer MeSa. Instead, in the course of selection the lines selected to avoid MeSa shifted their mean response towards a preference for MeSa. An inverse, albeit weaker, shift was detected for the lines selected to prefer MeSa. We discuss the factors that may have caused the apparent lack of a response to selection within isofemale line in this study and propose experimental approaches that address them.
\end{abstract}

Keywords Phytoseiulus persimilis · Methyl-salicylate · I-tube olfactometer · Genetic variation $\cdot$ Preference $\cdot$ Avoidance $\cdot$ Artificial selection

\section{Introduction}

Plants infested with herbivores release specific volatile compounds that are involved in the attraction of natural enemies of these herbivores (Dicke and Van Loon 2000; Sabelis et al.

B. Sznajder · M. W. Sabelis · M. Egas $(\bowtie)$

Department of Population Biology, Institute for Biodiversity and Ecosystems Dynamics, University of Amsterdam, Science Park 904, P.O.Box 94240, 1090 GE, Amsterdam, The Netherlands

e-mail: C.J.M.Egas@uva.nl 
2007). It is well established that the composition of the blends of volatiles depends on the species of herbivore (Sabelis and van de Baan 1983; De Moraes et al. 1998; Du et al. 1998) and the degree of infestation (Du et al. 1998; Maeda and Takabashi 2001; Nachappa et al. 2006). Therefore, the hypothesis was put forward that herbivore-induced plant volatiles may serve the natural enemies, such as predatory mites, as a signal of prey presence (Dicke and Sabelis 1988; Dicke and Van Loon 2000; Kessler and Baldwin 2001; Janssen et al. 2002). However, a single herbivore species may induce qualitatively and quantitatively different volatile blends on different plant species (van den Boom et al. 2004). Moreover, in natural settings the blends of volatiles produced by plants infested with various herbivore species will mix (Shröder and Hilker 2008). These results beg the question whether predators can extract a signal of prey presence from the variation in the blends of herbivore-induced plant volatiles. Adaptive learning may serve to update predator preferences for specific volatiles (or their mixtures) if these are experienced in the presence of suitable prey (Drukker et al. 2000; de Boer and Dicke 2004a, de Boer et al. 2005; Takabayashi et al. 2006). Moreover, a genetically determined response to specific volatiles may evolve by natural selection if these volatiles are consistently associated with the presence of suitable prey and genetic variation in predator response to these volatiles exists (Allison and Hare 2009).

We studied the response to selection imposed within iso-female lines established from a population of the predatory mite Phytoseiulus persimilis Athias-Henriot (Acari: Phytoseiidae) that was collected in the field. In particular, we asked whether it is possible to obtain lines of $P$. persimilis expressing contrasting responses to a specific plant volatile, methyl salicylate (MeSa). MeSa is consistently part of the blend of volatiles induced by feeding of the herbivorous mite Tetranychus urticae Koch (Acari: Tetranychidae) - the prey of P. persimilis - on a variety of plant species (van den Boom et al. 2004). Phytoseiulus persimilis is attracted to a range of concentrations of MeSa presented in pure form (Dicke et al. 1990; de Boer and Dicke 2004b; de Boer et al. 2004; van Wijk et al. 2008). Using gene-silencing techniques, Ament et al. (2010) dramatically reduced the emission of MeSa in tomato plants infested with T. urticae without changing the induced emission of the remaining volatiles and found that $P$. persimilis no longer differentiated between the infested and uninfested plants. Together, the evidence supports the hypothesis that the response of $P$. persimilis to MeSa may aid the predator in its search for prey and thus influence its fitness, although evidence for life-time fitness benefits under natural settings is yet to be shown. Furthermore, natural populations of $P$. persimilis harbour genetic variation in their response to MeSa when offered as a pure compound, against a background of volatiles from uninfested plants (Sznajder et al. 2010), as well as genetic variation in the response to the blends of plant volatiles induced by two-spotted spider mites on Lima bean plants (Margolies et al. 1997; Jia et al. 2002). Thus, there is potential for the evolution of predator responses to MeSa through natural selection.

We imposed purifying selection for contrasting responses of $P$. persimilis to MeSa when offered as a pure compound. The selection was performed on individuals within iso-female lines and two groups of iso-female lines were established where in one group the lines were propagated via females that showed a preference for MeSa, whereas in the second group the lines were propagated via females that avoided MeSa. This procedure aimed at setting apart and fixing genotypes distinct with respect to predator response to $\mathrm{MeSa}$ in genetically homozygous lines. Lines were thus purified in six rounds of selection, and the response to MeSa was measured after three and six rounds. 


\section{Materials and methods}

\section{Predators}

The base population and the iso-female lines of $P$. persimilis were maintained in a climate room $\left(25^{\circ} \mathrm{C}, 70 \%\right.$ humidity, and light:dark $\left.=16: 8\right)$ on a diet of $T$. urticae offered on detached leaves of Lima bean plants. Fresh leaves of Lima bean plants infested by twospotted spider mites were provided on a daily basis to each individual population. The base population originated from a sample of predators collected in 2002 at different locations throughout Sicily (Partinico, Scopella, Trappeto, Terrasini, Siculiana, Laghetto, Menfi, Trabia, Alcamo). Thus, it was presumably genetically variable, although local populations of $P$. persimilis are generally considered to be inbred under natural settings (Helle and Overmeer 1973).

Selection within iso-female line

Previous work (Sznajder et al. 2010) showed that variation among iso-female lines in their mean response to $\mathrm{MeSa}$ is greater when the predators are starved. Therefore, in this study the selection was conducted on starved predators. These were obtained by food deprivation applied for $24 \mathrm{~h}$ prior to a selection test. During the period of food deprivation females were kept in an Eppendorf vial at $18^{\circ} \mathrm{C}$ with water provided in a strip of wet filter paper. The vial had a gauze-covered opening to allow for airflow but was otherwise sealed.

Selection criteria were applied to individual females within iso-female lines. The isofemale lines were established by mated females selected from the base population in the first round of selection (a single binary choice-test for MeSa vs. clean air; the details of this choice-test follow in the next section) as illustrated in Fig. 1. Typically in haplodiploid arthropods such lines are obtained through mating between a virgin female and her sons. However, $P$. persimilis is pseudo-arrhenotokous, i.e. sons and daughters arise from fertilized eggs and male zygotes become haploid due to paternal genome inactivation and elimination during embryogenesis (Helle et al. 1978; Nelson-Rees et al. 1980). Hence, mating is a prerequisite for producing eggs destined to become females as well as males. Therefore, in order to establish iso-female lines we selected females that had already mated, instead of virgin females, and maintained these lines by brother-sister mating. By doing so we did not control for the genetic contribution of males to the next generation. However, assuming additive genetic variation for the selected trait and random mating, the confounding effect of the genetic contribution via unknown males was expected to be eradicated in the course of the selection rounds.

In the first selection round (Fig. 1) we established two groups of iso-female lines originating either from (1) the females that showed a preference for MeSa (15 lines, referred to as Plus lines) or (2) the females that showed an avoidance of MeSa (9 lines, referred to as Minus lines). The females selected in the first round are further referred to as founders of the iso-female lines (or founders for shorthand). Each iso-female line was further propagated via females whose responses were consistent with the response of the founder of their line. Propagation of the lines was performed on the basis of single choice-tests (see next section) and the females that made the same choice as the founder of their line were placed each in a separate Petri dish to establish descendant iso-female lines. We imposed such bottlenecks within each line at intervals of about 2.5 weeks. The developmental time from egg to adult in $P$. persimilis is about 7 days (Sabelis 1981) and a female lays about 3 eggs per day under laboratory conditions prevailing in this study. Thus, by applying selection round every 


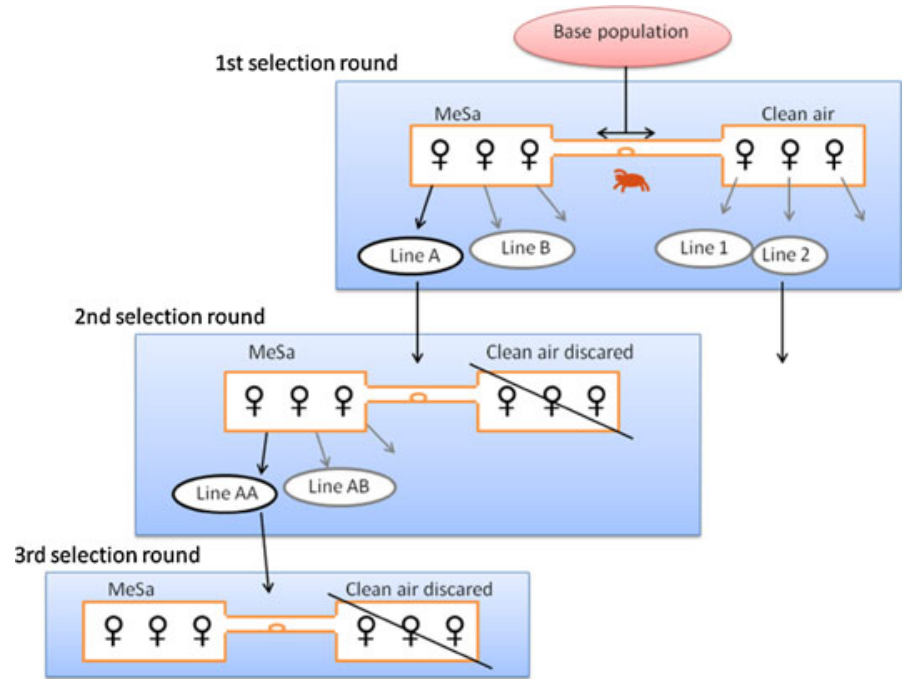

Fig. 1 Selection within iso-female lines is illustrated on the example of the single line A and its descending lines during the first three selection episodes (see Selection within iso-female line for details). Note that the label of the iso-female line carries information on relatedness among the lines, i.e. first letter/number indicates the identity of the female selected in the first round of selection (i.e. founder), second letters/ numbers indicate the identity of her daughters selected to continue the line by each establishing a new line, and so on

2.5 weeks we ensured there were at least about 10 mated females available for continued selection on each iso-female line.

In total we imposed six rounds of selection. However, using the above procedure the number of lines would grow exponentially with every selection round (even though some lines failed to establish). Therefore, after three selection rounds we temporarily halted the experiment in order to evaluate the responses of already established lines. The mean responses of these lines were evaluated relative to the mean response of the base population measured at the start of the selection experiment. Selection was continued in Plus lines where the mean proportion of individuals choosing MeSa was not lower than in the base population (i.e. 0.64), and in Minus lines where the mean proportion was not higher than in the base population. Based on this criterion we resumed selection in four Plus lines and in three Minus lines. Thus, after another three rounds of selection we established 19 Plus lines and 14 Minus lines.

Genetic exchange among the iso-female lines was prevented by maintaining each line in a sealed large Petri dish with a small opening covered with gauze (mesh width $=0.07 \mathrm{~mm}$, average adult predator size $=0.5 \mathrm{~mm}$, average predator egg size $=0.2 \mathrm{~mm}$, thus the mesh width is small enough to prevent immigration and emigration) to allow for airflow. A rearing was accessed by opening the Petri dish on an alcohol-cleaned surface. Prior to opening and after re-sealing, the outside surface of the Petri dish was cleaned with an alcohol-soaked cloth.

Olfactory choice-test

We used a choice-test between MeSa in pure form and clean air to (1) select individual females with respect to their response to MeSa within iso-female lines, and to (2) evaluate 
the mean responses of these lines after three and six rounds of selection. We used a single choice-test to determine the behaviour of the predator in order to exclude the possibility that the experience with the presentation of MeSa in the experimental set-up influenced its response to MeSa. Individuals that chose to walk towards the clean air source were interpreted as avoiding $\mathrm{MeSa}$, while individuals that chose to walk towards the MeSa source were interpreted as having a preference for MeSa. The responses of the iso-female lines were calculated as the mean proportion of females choosing MeSa (the number of adult females per replicate experiment varied between 17 and 25).

The choice-test was conducted in a so-called I-tube olfactometer: a single straight glass tube (length $20 \mathrm{~cm}, \varnothing 0.5 \mathrm{~cm}$ ) with a small opening $(2 \mathrm{~mm})$ in the middle to introduce the predatory mites (here we give an abbreviated description of this setup; for a full description see Sznajder et al. 2010). This olfactometer was provided with a trap at the end of either arm of the I-tube to collect predators. Opposite the entrance hole, another opening $(\varnothing 0.5 \mathrm{~cm})$ was present which was gauze-covered and served as an air-outlet. At the ends of the I-tube there were plastic trap vials $(\varnothing 30 \mathrm{~mm}$, height $55 \mathrm{~mm})$ which in turn were connected to jars that either contained a capillary with MeSa or not. The traps were designed as an easy-to-enter-yet-difficult-to-exit vial, and were provided with a water source (wet cotton wool). After purification by activated carbon filters, air was flowing at $20 \mathrm{l} / \mathrm{h}$ through the jars, the vials and the arms of the glass tube, leaving the system through the opening in the middle of the I-tube. At release in the middle of the I-tube, a predatory mite finds itself in air streams coming from right and left, i.e. one with $\mathrm{MeSa}$ and the other without. Subsequently, it can move left or right in the I-tube and ultimately it enters one of the trap vials or it stays in the I-tube. After release of the test animals, the entrance hole was sealed with Parafilm ${ }^{\circledR}$. Pilot experiments showed that the I-tube olfactometer produces results consistent with our knowledge of the responses of $P$. persimilis to herbivoreinduced plant volatiles (Van Wijk, unpublished data).

Within a single replicate experiment a group of females was released sequentially into the I-tube, i.e. the individuals were released in the olfactometer one after the other. Visual cues play no role in predator orientation as the predator is blind and orients itself by means of chemical cues. Previous studies using a Y-tube set-up showed that there is no effect of possible residues deposited along the path taken by an individual on the choices of subsequent individuals (Sabelis and van de Baan 1983; van Wijk et al. 2010). After 25 min, the number of mites in each of the two trap vials was counted. The number that remained in the I-tube, was scored as "no choice". For each replicate of a line, a new clean I-tube was used and the side of the arm containing air with MeSa was interchanged to exclude any effects of unforeseen asymmetries in the experimental set-up.

Synthetic MeSa (Sigma-Aldrich Fluka, pure; assay $\geq 99 \%$ ) was offered undiluted in a small capillary $(9 \mu \mathrm{l}, \varnothing 0.60 \mathrm{~mm}$, Omnilabo) placed in one of the jars connected to the I-tube. The air flow through the set-up was started at least $2 \mathrm{~h}$ before the start of the test. The MeSa evaporation rate was $\pm 30 \mu \mathrm{g} / \mathrm{h}$. This evaporation rate was chosen based on the responses elicited in the general population. In particular, in order to increase our chances of detecting selection for both and increased and decreased response to MeSa we used the concentration that elicits neutral responses (50:50) in the base population.

Statistical analysis

We hypothesized that the propagation of iso-female lines through individuals that exhibited either preference for MeSa or aversion to MeSa would lead to a shift in the mean responses of those lines towards, respectively, preference for MeSa or aversion to MeSa. 
Fig. 2 The responses of the iso-female lines selected to prefer MeSa (labelled with letters) and the lines selected to avoid $\mathrm{MeSa}$ (labelled with numbers) obtained after a three and $\mathbf{b}$ six rounds of selection. The empty circle represents the proportion of individuals choosing MeSa per replicate $(\mathrm{N}=15-25$ individuals) calculated excluding the no-choice individuals. The filled circles indicate the mean proportions of individuals choosing MeSa calculated per iso-female line. The dotted vertical line indicates the response of the base population at the start of the selection. Note that not all the lines presented in Fig. a were selected past the third selection round (see Selection; Fig. 3). Note that the relatedness among the lines is indicated by the labels of the lines (see also Fig. 1). Hence, e.g. the lines FAA FAC and FBA share their great grandmother $\mathrm{F}$ (i.e. the founder), and the lines $F A A$ and $F A C$ share also their grandmother A

Therefore, we asked whether there was a significant difference in the mean response to MeSa between the Plus lines and the Minus lines after three and (or) six rounds of selection. Moreover, we wished to make inferences about the sources of random variation due to iso-female line. To this end, we constructed a mixed-effects model (Pinheiro and Bates 2000; Venables and Ripley 2002) that included the fixed effects of (1) the selection treatment (two levels: selection for a preference for MeSa, selection for an aversion to $\mathrm{MeSa}$ ), (2) the time point at which the average responses of the iso-female lines were determined (two levels: after the 3rd round of selection, after the 6th round of selection) and (3) the proportion of no-choice individuals as a covariate. The model assesses the effect of these factors on the proportion of individuals choosing MeSa calculated for each replicate. The random effect consisted of the factor iso-female line nested in the factor founder (where founder was the female selected in the first round of selection, see Selection). Such a grouping structure reflected relatedness among the iso-female lines as determined by the first common founder of these lines.

The diagnostics plots for the untransformed response variable showed that the assumptions of the mixed-effects model were satisfied (the random variables were normally distributed with mean zero and independent for different groups, and the withingroup errors were independent and normally distributed around a mean of zero). For this reason the analysis was performed on untransformed data, using SPlus 6.2 software (Pinheiro and Bates 2002). The significance of the fixed effects, their interaction and the significance of the random effect was tested using likelihood ratio tests (Pinheiro and Bates 2000; Venables and Ripley 2002).

\section{Results}

The behaviour of the base population did not change during the selection experiment. The mean proportion of individuals choosing MeSa was 0.64 at the start $(\mathrm{SE}=0.15, \mathrm{~N}=57$ ) as well as at the end of the selection experiment $(\mathrm{SE}=0.13, \mathrm{~N}=101)$. In contrast, after three rounds of selection the mean responses of the selected iso-female lines were variable and include preference and aversion in both the Plus and the Minus lines (Fig. 2a). This variation among the iso-female lines was slightly lower after six rounds of selection (Fig. 2b). However, the variation in the mean responses of the Minus lines was shifted in the direction opposite to the selected criterion, as compared to the mean responses shown by their ancestral lines after three rounds of selection.

We did not find a significant difference in the mean responses to MeSa between the Plus and Minus lines (likelihood ratio test $P_{\text {selection }}=0.09$, Table 1). This was the case after three rounds of selection as well as after six rounds of selection (likelihood ratio test $P_{\text {time- }}$ point $=0.21$, Table 1 ). However, we found an interaction between the selection and the time-point at which the responses of the lines were measured (likelihood ratio test, 
(a)

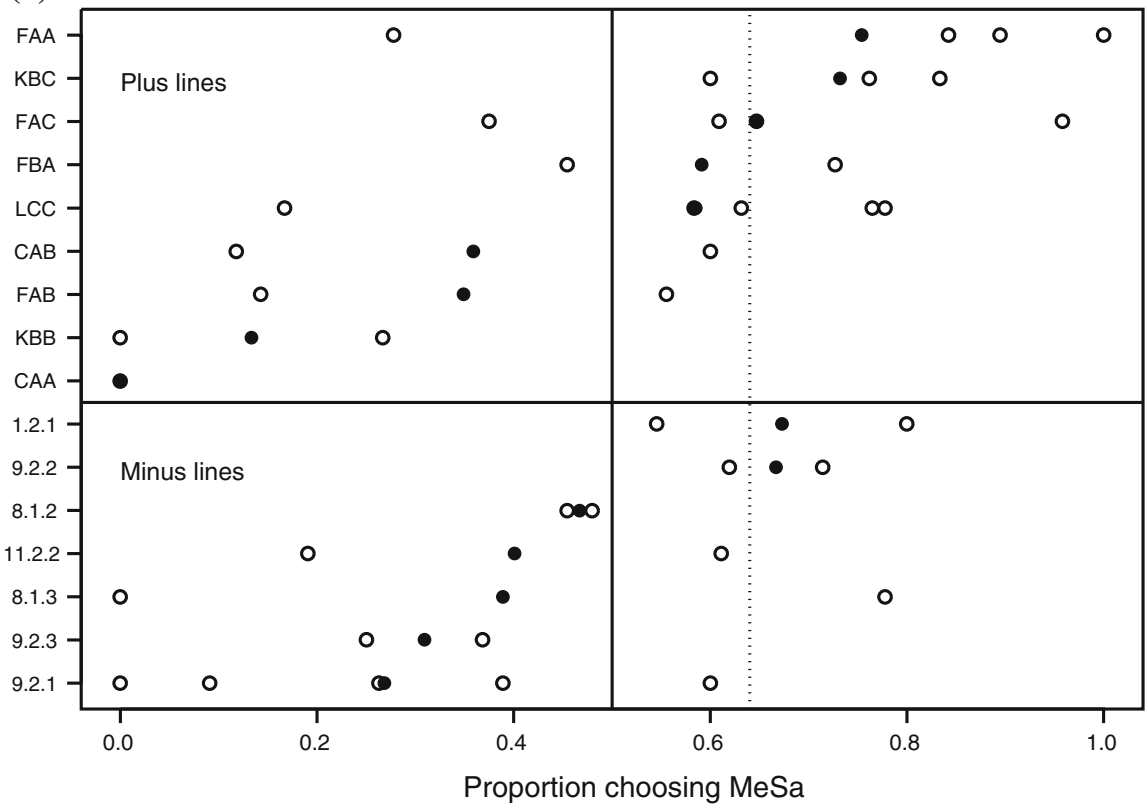

(b)

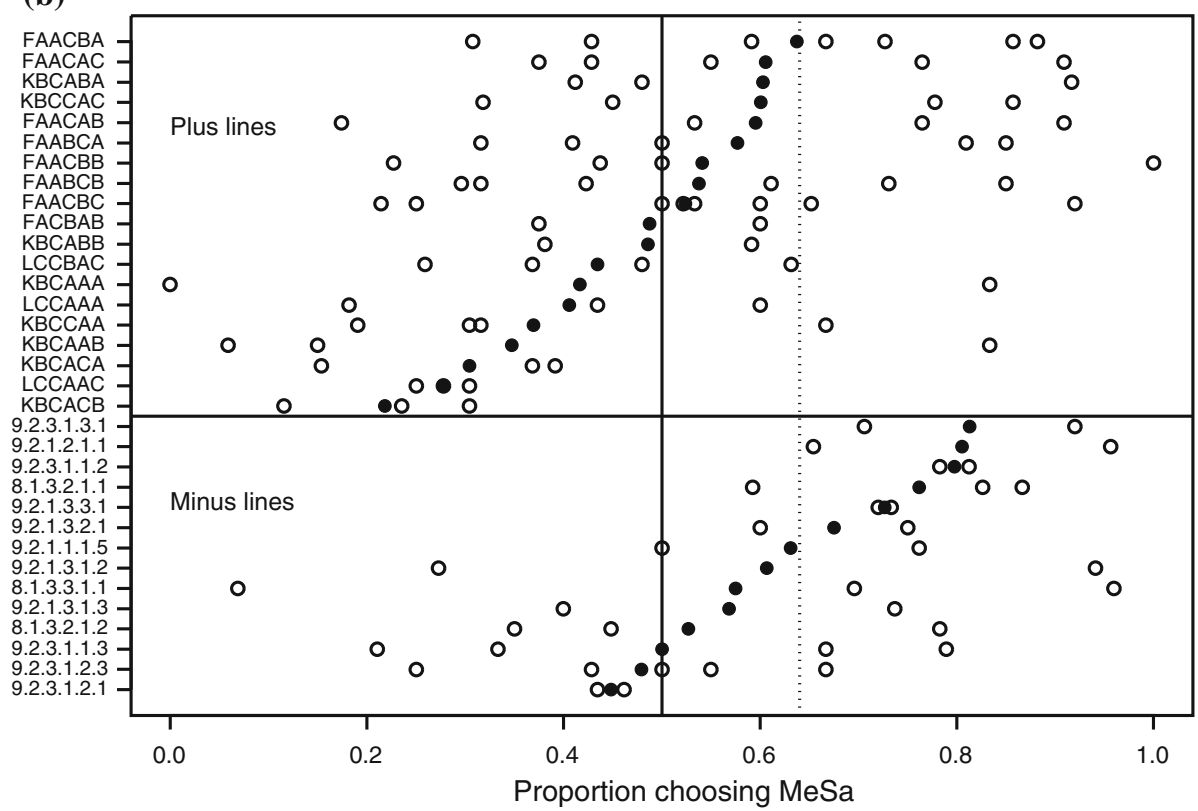

$P_{\text {selection:time-point }}=0.02$, Table 1 ). Given the non-significant effect of the selection and the time-point factors, this interaction indicates that there was a significant difference between the Plus and Minus lines at the end of the selection (but not half-way through the selection) and this difference was due to a change in behaviour of the Minus lines but not the Plus 
Table 1 Mixed-effects model of predator response to MeSa in the two-choice test: MeSa versus clean air

\begin{tabular}{llll}
\hline Effect & Likelihood ratio $\left(d f_{1}, d f_{2}\right)$ & SD & $P$ value \\
\hline Random & & & 0.08 \\
$\quad$ Founder & $(9,8)$ & 0.0004 & 0.02 \\
Iso-female line & $(9,8)$ & 0.29 & 0.09 \\
Residual & & & 0.21 \\
Fixed & $(8,7)$ & & 0.25 \\
Selection treatment & $(8,7)$ & & 0.02 \\
$\quad$ Time point & & & \\
Covariate & $(8,7)$ & & \\
$\quad$ Proportion no choice & $(9,8)$ & & \\
Interaction & & & \\
Selection treatment:time point & & & \\
\hline
\end{tabular}

The random effect consists of the iso-female line nested in the founder. Fixed effects include the selection (levels: Plus, Minus), the number of selection rounds imposed on the lines (levels: three, six) and the proportion of individuals making no choice added as covariate. The amount of variation explained by grouping with respect to founder, iso-female line and the residual variance is presented in the form of standard deviations $(S D)$

lines. Figure 2 reveals that the change in the response of the Minus lines was opposite to the direction selected. After six rounds of selection the Minus lines shifted their average responses towards a preference for $\mathrm{MeSa}$, when compared to the responses of their ancestor lines measured after three episodes of selection.

The analysis was performed on data including the responses of the iso-female lines that were not subject to selection past the third round (see Selection and Statistical analysis). In order to further explore the responses of the lines as a function of the behaviour of their ancestor lines we plotted the mean responses of the lines obtained after six rounds of selection as a function of the behaviour of their ancestor lines that were measured after three rounds of selection. This relationship is presented in Fig. 3 showing a shift in mean responses of the Minus lines but also an equivalent shift in the responses of the Plus lines (albeit weaker). This result for the Plus lines was not detected in the above analysis using the mixed-effects model presumably due to the greater variation among the Plus lines.

We found a significant effect of founder (likelihood ratio test $P_{\text {founder }}=0.02$, Table 1 ), i.e. the grouping with respect to founder (between-founder variation) explained a significant amount of variation in the behaviour of the lines. This confirms that there was genetic variation in predator responses to $\mathrm{MeSa}$ in the base population and the founder effect indicates a genetic background that was "frozen" within the iso-female lines at the start of the selection experiment. Adding the iso-female line effect (nested in the founder effect) did not explain more variation in predator responses to MeSa (likelihood ratio test $P_{\text {line }}=0.10$, Table 1 ). The variation explained by the iso-female line effect was smaller (by orders of magnitude) than the variation explained by the founder effect (Table 1). The iso-female line effect represents the deviation in the behaviour of individual lines from the mean response of the group of lines that shared a founder (within-founder variation). Thus, the non-significant effect of the iso-female line indicates that there was little withinfounder variation among the lines while controlling for the effect of the main factors selection treatment and time point. Moreover, we observed a relatively large amount of residual variance that represents the deviation of the individual replicates from the average response of the iso-female lines (the within-line variability, Table 1). Thus, there was a 


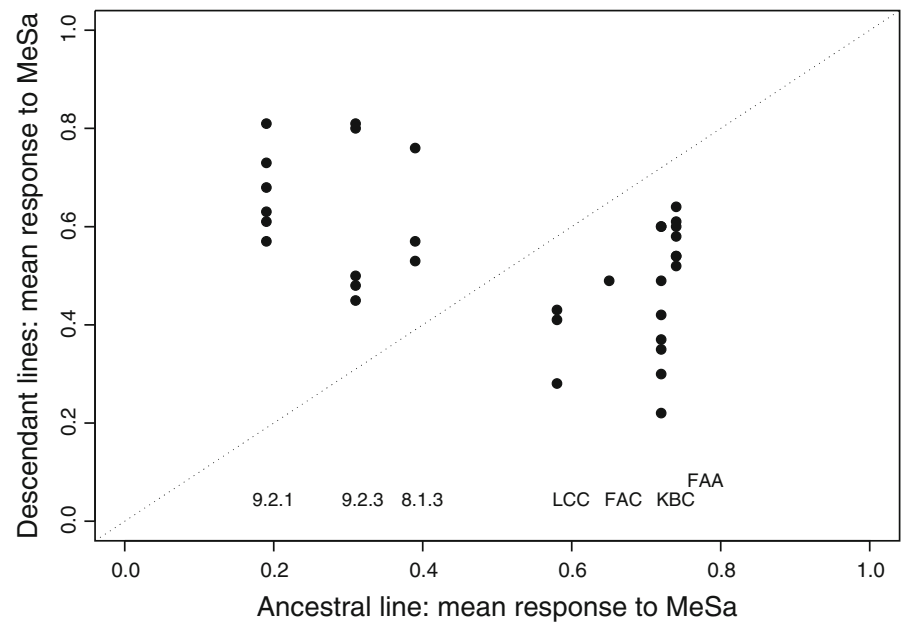

Fig. 3 Mean responses of the descendant lines after six rounds of selection (y-axis) as a function of the responses of their ancestral lines after three rounds of selection (x-axis). Only the names of the ancestral lines are shown; letter labels refer to the lines selected for the preference of MeSa (Plus lines) while number labels refer to the lines selected for the aversion of MeSa (Minus lines). The circles represent the mean proportions of individuals choosing $\mathrm{MeSa}$ calculated per line (for the responses of individual replicates see Fig. 2). Successful directional selection for extreme responses to $M e S a$ would result in a positive correlation between these proportions such that the descendant Plus lines would lie either on or above the dotted diagonal line, while the descendant Minus lines would lie on or below the diagonal line

substantial environmentally determined component in predator responses to $\mathrm{MeSa}$ as measured in the olfactory test used in this study.

The average proportion of individuals not making a choice amounted to 0.16 . The covariate representing the proportion of individuals that made no choice in the olfactory test was not significant (likelihood ratio test $P_{\text {proportion no-choice }}=0.25$, Table 1 ), i.e. there was no relationship between the mean response to MeSa in a single replicate test and the number of individuals that made no choice in this replicate.

\section{Discussion}

We showed that the amount of variation in predator responses to Mesa explained by the founders of the iso-female lines was significantly different from zero, thus confirming that the responses of $P$. persimilis to MeSa have a genetic component (Sznajder et al. 2010). However, we have not found expected differences in the behaviour between the iso-female lines maintained via individuals that chose MeSa (Plus lines) and the lines maintained via the individual that avoided MeSa (Minus lines). Instead, we observed a shift in the mean response to $\mathrm{MeSa}$ in the direction opposite to the selection criterion, i.e. the responses of the Minus lines at the end of selection were significantly shifted towards preference of $\mathrm{MeSa}$ as compared to the responses of their ancestor lines half way through the selection (Fig. 3). The corresponding pattern, albeit weaker, was observed for the Plus lines.

Our experiment aimed at purifying the responses of individual lines and to this end we repeatedly re-established the lines via single mated females that expressed the behaviour in question (i.e. either preference of $\mathrm{MeSa}$ or avoidance of MeSa). Therefore, this procedure led to obtaining genetically homozygous lines. Such lines can be further used to study the 
genetic determination of predator response to $\mathrm{MeSa}$; the analysis of reciprocal crosses between iso-female lines test, for example, whether more extreme responses to MeSa can be observed in heterozygous genotypes. Inbreeding in the lines of $P$. persimilis was less likely to be associated with inbreeding depression because in haplodiploids deleterious alleles are immediately expressed in haploid males and therefore can be purged by natural selection (Crozier 1985; Werren 1993; Henter 2003; Charlesworth and Willies 2009, unless these alleles have female-limited effect; Saito et al. 2001; Tien et al. 2010 submitted).

Re-establishing the lines via single mated females creates conditions favourable for random genetic drift. If in this study random genetic drift was stronger than selection within iso-female line then the genetic differences between and within the Plus and Minus lines would become fixed by chance. In particular, random genetic drift is expected to overcome selection if $\mathrm{N}_{\mathrm{e}} \mathrm{s}<1$, i.e. the product of the effective population size and selection coefficient is less than unity (Hartl and Clark 1989). To illustrate this relationship, suppose that predator response to MeSa is determined by a single gene that has two alleles: an allele for preference of MeSa and an allele for the avoidance of MeSa. Hence, in this study selection would be effective if the selection coefficient was $s>0.67$ (given that the population is started by a single mated female and $\mathrm{N}_{\mathrm{e}}$ in a haplo-diploid system is given by $\left(9 * N_{f} N_{m}\right) /\left(2 N_{f}+4 N_{m}\right)$; Hedrick and Parker 1997). In this experiment, in the lines selected for preference of MeSa, the genotypes that showed avoidance of MeSa were eliminated, hence their fitness was assumed to be $W=0$, and their selection coefficient $\mathrm{s}=1$ (in the lines selected for avoidance of MeSa the selection coefficient of the genotype showing preference for MeSa $s=1$ ). In reality the selection was performed on phenotypes in a single selection test and it cannot be expected that these phenotypes always reflected their genotypes. Therefore, the correct genotypes may not have been always selected for, and in such situations the selection coefficient of the out-selected genotypes would have been less than one. However, in this theoretical system, for the selection coefficient to be lower than 0.67 the rate of selecting a phenotype inconsistent with its genotype (e.g. a phenotype that shows preference of MeSa while its genotype would avoid MeSa) must be equal to or more than $0.33\left(\mathrm{~s}=1-\mathrm{W}=1-P_{\mathrm{a}} / P_{\mathrm{A}}\right.$, where $\mathrm{P}_{\mathrm{X}}$ is the probability of genotype $\mathrm{X}$ contributing to the next generation). This means that in about $67 \%$ of selected cases the phenotype reflects its genotype but in $33 \%$ of these cases it does not.

The above analysis serves as an illustration only because the details of the genetic architecture of the response to MeSa in P. persimilis are not know; in fact this study is among the first (Sznajder et al. 2010) to provide evidence that there is a genetic basis for this behaviour (for similar findings in the predatory mite Neoseiulus womersleyi, see Maeda 2006). Therefore, the number of loci influencing this trait (and hence the appropriateness of the above model) or the magnitude of the error in selecting wrong genotypes based on their phenotypes are yet unknown. Thus, we cannot exclude the possibility that random genetic drift may have played a role in some of the selection episodes.

It is informative to discuss other factors that may have contributed to the differences in the average responses to MeSa among the Plus and Minus lines in this experiment. In particular, the genetic component for a trait inferred from the differences among iso-female lines [as in this study and in Sznajder et al. (2010)] comprises additive as well as nonadditive genetic effects. This role of non-additive versus additive effects in the genetic determination of predator response to MeSa may be explored by analysis of reciprocal crosses between iso-female lines that are different with respect to this trait (e.g. Carton et al. 1992).

For the correct interpretation of a change in the behaviour of iso-female lines due to an experimental treatment the influence of general environmental effects that pertain to 
common rearing conditions must be minimised (David et al. 2005). However, in order to explain the results of this study, such general environmental effects would have to influence the Plus lines and Minus lines in a consistently different way. Moreover, their effect would have to change between the mid-point (after three rounds of selection) and the endpoint (after six rounds of selection) of the experiment. Finally, environmental effects would most likely be also reflected in a shift of the mean response of the base population (as this population was maintained in the same climate room as the selection lines but physically separated). The mean responses to MeSa of the base population, however, did not change as measured between the start and the end of the selection experiment (although fluctuations in the responses of the base population in-between the measurements cannot be ruled out). Based on these results we conclude that general environmental effects may have played only a minor role.

In this study the selection was performed on mated females, i.e. for practical considerations the females were allowed to mate in the culture and, thus, prior to the selection test. Therefore, the responses to MeSa of the males were not subject to selection and the genetic contribution of the males to the next generation was not controlled. Assuming additive genetic variance for the selected trait and random mating this effect of uncontrolled genetic contribution of the males should be eradicated in the course of selection rounds. Suppose, however, that mating is non-random such that, for example, females that show preference for MeSa mate more often with males that avoid MeSa. The resulting disassortative mating may lead to responses of the selected lines fluctuating within the spectrum between preference and aversion as observed in different generations of our selection experiment. Any form of non-random mating would hinder directional selection and it may possibly be realized with respect to a trait correlated with olfactory responses, rather than with respect to the response to $\mathrm{MeSa}$ itself. There is evidence of non-random mating in $P$. persimilis. Virgin females were shown to mate more readily with unrelated mates in no-choice mating experiments (although no such preference for non-kin mates was found in choice experiments, Enigl and Schausberger 2004). Moreover, P. persimilis may be able to recognize individuals based on familiarity, as shown for juvenile predators (Schausberger 2005, 2007). A relationship between mating preference and olfactory responses of the partners could provide an explanation for the observed shift in the behaviour of the selected lines in our experiment. If such a relationship holds, then selection on the olfactory responses of $P$. persimilis requires that the mating is ensured to take place among individuals exhibiting the same olfactory response. Uncovering any such relationship requires that we study the olfactory responses of males as well as females and their mating preferences with respect to this behaviour.

$\mathrm{MeSa}$ presented in a pure form without a background of volatiles was a novel experience to the predators in the sense that the predators had not perceived it prior to the selection test. However, the predators were reared on detached leaves of plants infested with $T$. urticae. Therefore, they had experience with the full blend of plant volatiles released by $T$. urticae-infested plants and this full blend does include MeSa. This experience may influence predator responses in selection, however evidence to date shows that predator responses to pure compounds does not explain subsequent responses to mixtures of these compounds and, conversely, predator attraction to the full volatile blend induced by $T$. urticae is not due to attraction to all individual compounds constituting this blend (van Wijk 2007; van Wijk et al. 2008). There is evidence that experience acquired during rearing on infested plants may influence subsequent predator responses to full volatile blends of these plants when uninfested (Takabayashi and Dicke 1992, but see also van den Boom et al. 2002 for no effect of rearing history on predator responses to full 
blends). In general, exposing P. persimilis to full blends of volatiles (Drukker et al. 2000, de Boer and Dicke 2004a, de Boer et al. 2005, van Wijk 2007) or individual volatile compounds (Sznajder et al. unpublished manuscript) that are coupled with the presence of $T$. urticae resulted in increased predator preference for these compounds. In line with these results, suppose that prior experience of predators with the full blend of volatiles did increase predator responsiveness to $\mathrm{MeSa}$ in the selection test. In this scenario, predators that showed a preference for MeSa may have acquired this behaviour due to learning. Therefore, the selection would not change the mean behaviour of the lines established from selecting such individuals (because the learned responses masked the genetically determined behaviour and no change in the mean trait value between generations could be obtained). Alternatively, the selection could further increase the preference of MeSa in those lines because the set-up would effectively select for improved learning of MeSa, provided that there is genetic variation in learning ability in this population. Furthermore, according to this hypothesis, selected predators that avoided MeSa should be interpreted as poor learners presumably revealing their genetically determined response to MeSa. Hence, selection via these individuals would lead to stronger aversion to MeSa exhibited in the descendant selection lines. As can be seen in Fig. 3, these predictions are not consistent with the results of this study. Although we cannot exclude the possibility that the experience prior to the selection tests did not influence the predator responses, current knowledge on how experience shapes predator responses to herbivore-induced plant volatiles does not provide a straightforward explanation for the results obtained in this study.

Finally, the role of maternal effects shaping predator responses to MeSa (i.e. nonMendelian transmission of a trait from mother to offspring) was not addressed in this study. Maternal effects may influence the magnitude as well as the direction of population responses to selection (Kirkpatrick and Lande 1989, Lande and Kirkpatrick 1990). They encompass a diversity of effects deriving from parental influence during rearing (various forms of parental care or the choice of oviposition sites) or cytoplasmic effects pertaining to inheritance of cytoplasmic factors passed on to the egg during oogenesis. To the best of our knowledge there is no parental care in $P$. persimilis, and an effect of oviposition in an environment characterized by different volatile profiles can also be excluded (as all lines were reared on Lima bean leaves infested with T. urticae). However, we cannot rule out a cytoplasmic maternal effect.

In conclusion, our results confirm that the responses of P. persimilis to MeSa have a genetic component. Future studies should address the response to MeSa (or other herbivore-induced plant volatiles) of males as well as females and thus help explain whether any correlation between predator mating preferences and the response to MeSa may contribute to the outcome of selection.

Acknowledgments We thank Tadeusz Kawecki for his critical comments on an earlier draft of this manuscript, as well as two anonymous reviewers.

Open Access This article is distributed under the terms of the Creative Commons Attribution Noncommercial License which permits any noncommercial use, distribution, and reproduction in any medium, provided the original author(s) and source are credited.

\section{References}

Allison JD, Hare JD (2009) Learned and naïve natural enemy responses and the interpretation of volatile organic compounds as cue or signals. New Phytol 184:768-782 
Ament K, Krasikov V, Allmann S, Rep M, Takken FLW, Schuurink RC (2010) Methyl salicylate production in tomato affects biotic interactions (in press)

Carton Y, Frey F, Nappi A (1992) Genetic determinism of the cellular immune reaction in Drosophila melanogaster. Heredity 69:393-399

Charlesworth D, Willies JH (2009) The genetics of inbreeding depression. Nat Rev Genet 10:783-789

Crozier RH (1985) Adaptive consequences of male-haploidy. In: Helle W, Sabelis MW (eds) Spider mites, their biology, natural enemies and control. Elsevier Science Publishers, Amsterdam

David JR, Gibert P, Legout H, Pètavy G, Capy P, Moreteau B (2005) Isofemale lines in Drosophila: an empirical approach to quantitative trait analysis in natural populations. Heredity $94: 2-12$

De Boer JG, Dicke M (2004a) Experience with methyl salicylate affects behavioural responses of a predatory mite to blends of herbivore-induced plant volatiles. Entomol Exp Appl 110:181-189

De Boer JG, Dicke M (2004b) The role of methyl salicylate in prey searching behaviour of the predatory mite Phytoseiulus persimilis. J Chem Ecol 30:255-271

De Boer JG, Posthumus MA, Dicke M (2004) Identification of volatiles that are used in discrimination between plants infested with prey and nonprey herbivores by a predatory mite. J Chem Ecol 30:2215-2230

De Boer JG, Snoeren TAL, Dicke M (2005) Predatory mites learn to discriminate between plant volatiles induced by prey and nonprey herbivores. Anim Behav 69:869-879

De Moraes CM, Lewis WJ, Pare PW, Alborn HT, Tumlinson JH (1998) Herbivore-infested plants selectively attract predators. Nature 393:570-573

Dicke M, van Loon JJA (2000) Multitrophic effects of herbivore-induced plant volatiles in an evolutionary context. Entomol. Exp. Appl. 97:237-249

Dicke M, van Beek TA, Posthumus MA, Dom NB, van Bokhoven H, de Groot AE (1990) Isolation and identification of volatile kairomone that affects acarine predator-prey interactions: involvement of host plant in its production. J Chem Ecol 16:381-396

Drukker B, Bruin J, Sabelis MW (2000) Anthocorid predators learn to associate herbivore-induced plant volatiles with presence or absence of prey. Physiol Entomol 25:260-265

Du Y, Poppy GM, Powell W, Pickett JA, Wadhams LJ, Woodcock CM (1998) Identification of semiochemicals released during aphid feeding that attract parasitoid Aphidius ervi. J Chem Ecol 24: 1355-1368

Enigl M, Schausberger P (2004) Mate choice in predaceous mite Phytoseiulus persimilis: evidence of selfreferent phenotype matching? Entom Exper Appl 112:21-28

Hartl DL, Clark AG (1989) Principles of population genetics. Sinauer Associates, Sunderland

Hedrick PW, Parker JD (1997) Evolutionary genetics and genetic variation of haplodiploids and X-linked genes. Annu Rev Ecol Syst 28:55-83

Helle W, Overmeer WPJ (1973) Variability in tetranychid mites. Annu Rev Entomol 18:97-120

Helle W, Bolland HR, Van Arendonk R, de Boer R, Schulten GGM, Russell VM (1978) Genetic evidence for biparental males in haplo-diploid predator mites (Acaria: Phytoseiidae). Genetica 49:165-171

Henter JJ (2003) Inbreeding depression and haplodiploidy: Experimental measures in a parasitoid and comparison across diploid and haplodiploid insect taxa. Evolution 57:1793-1803

Janssen A, Sabelis MW, Bruin J (2002) Evolution of herbivore-induced plant volatiles. Oikos 97:134-138

Jia F, Margolies DC, Boyer JE, Charlton RE (2002) Genetic variation among foraging traits in inbred lines of a predatory mites. Heredity 88:371-379

Kessler A, Baldwin IT (2001) Defensive function of herbivore-induced plant volatile emissions in nature. Science 291:2141-2144

Kirkpatrick M, Lande R (1989) The evolution of maternal characters. Evolution 43:485-503

Lande R, Kirkpatrick M (1990) Selection response in traits with maternal inheritance. Genet Res Camb 55:189-197

Maeda T (2006) Genetic variation in foraging traits and life-history traits of the predatory mite Neoseiulus womersleyi (Acari: Phytoseiidae) among isofemale lines. J Insect Behav 19:573-589

Maeda T, Takabashi J (2001) Production of herbivore-induced volatiles and their attractiveness to Phytoseiulus persimilis (Acari: Phytoseiide) with changes of Tetranychus urticae (Acari: Tetranychidae) density on a plant. Appl Entomol Zool 36:47-52

Margolies DC, Sabelis MW, Boyer JE (1997) Response of a phytoseiid predator to herbivore-induced plant volatiles: selection on attraction and effect on prey exploitation. J Insect Behav 10:695-709

Nachappa P, Margolies DC, Nechols Jr, Loughin T (2006) Phytoseiulus persimilis response to herbivoreinduced plant volatiles as a function of mite-days. Exp Appl Acarol 40:231-239

Nelson-Rees WA, Hoy MA, Roush RT (1980) Heterochromatinization, chromatine elimination and haploidization in the parahaploid mite Metaseiulus occidentalis (Nesbitt) (Acarina: Phytoseiidae). Chromosoma 77:263-276 
Pinheiro JC, Bates DM (2000) Mixed-Effects models in S and S-PLUS. Statistics and Computing Series. Springer, New York

Sabelis, MW (1981) Biological control of two-spotted spider mites using phytoseiid predators. Agricultural research reports 910 Pudoc, Wageningen

Sabelis MW, Van De Baan HE (1983) Location of distant spider-mite colonies by phytoseiid predators: demonstration of specific kairomones emitted by Tetranychus urticae and Panonychus ulmi (Acari: Phytoseiidae, Tetranychidae). Entomol Exp Appl 33:303-314

Sabelis MW, Takabayashi J, Janssen A, Kant MR, Van Wijk M, Sznajder B, Aratchige NS, Lesna I, Belliure B, Schuurink RC (2007) Ecology meets plant physiology: Herbivore-induced plant responses and their indirect effects on arthropod communities, pp 188-217. In: Oghushi T, Craig TP, Price PW (eds) Ecological communities: plant mediation in indirect interaction webs. Cambridge University Press, Cambridge

Saito Y, Sahara K, Mori K (2001) Inbreeding depression by recessive deleterious genes affecting female fecundity of a haplo-diploid mite. J Evol Biol 13:668-678

Schausberger P (2005) Predatory mite Phytoseiulus persimilis manipulates imprinting among offspring through egg placement. Behav Ecol Sociobiol 58:53-59

Schausberger P (2007) Kin recognition by juvenile predatory mites: prior association or phenotype matching? Behav Ecol Sociobiol 62:119-125

Shröder R, Hilker M (2008) The relevance of background odor in resource location by insects: a behavioural approach. Bioscience 58:308-316

Sznajder B, Sabelis MW, Egas M (2010) Response of predatory mites to a herbivore-induced plant volatile: genetic variation for context-dependent behaviour. J Chem Ecol 36:680-688

Takabayashi J, Dicke M (1992) Response of predatory mites with different rearing histories to volatiles of uninfested plants. Entomol Exp Appl 64:187-197

Takabayashi J, Sabelis MW, Janssen A, Shiojiri K, van Wijk M (2006) Can plants betray presence of multiple herbivore species to predators and parasitoids? The role of learning in phytochemical information networks. Ecol Res 21:3-8

Tien NSH, Sabelis MW, Egas M (2010) Inbreeding depression and purging in a haplodiploid: gender-related effects. In: Tien, NSH, Evolutionary genetics of life-history traits in a haplodiploid mite. PhD thesis, University of Amsterdam

Van den Boom CEM, van Beek TA, Dicke M (2002) Attraction of Phytoseiulus persimilis (Acari: Phytoseiidae) towards volatiles from various Tetranychus urticae-infested plant species. Bull Entomol Res 92:539-546

Van Den Boom CEM, Van Beek TA, Posthumus MA, De Groot Æ, Dicke M (2004) Qualitative and quantitative variation between volatile profiles induced by Tetranychus urticae feeding on different plants of various families. J Chem Ecol 30:69-89

Van Wijk M (2007) Deciphering the code of herbivore-induced plant odours: the whole is different from the sum of its parts. Dissertation, University of Amsterdam

Van Wijk M, de Bruijn PJA, Sabelis MW (2008) Predatory mite attraction to herbivore-induced plant odors is not a consequence of attraction to individual herbivore-induced plant volatiles. J Chem Ecol 34:791-803

Van Wijk M, de Bruijn PJA, Sabelis MW (2010) The predatory mite Phytoseiulus persimilis does not perceive odor mixtures as strictly elemental objects. J Chem Ecol 36:1211-1225

Venables WN, Ripley BD (2002) Modern applied statistics with S, 4th edn. Springer, New York

Werren JH (1993) The evolution of inbreeding in halodiploid organisms. In: Thornhill NW (ed) The natural history of inbreeding and outbreeding. The University of Chicago Press, Chicago 